\title{
A Revisit to CMFD Schemes: Fourier Analysis and Enhancement
}

\author{
Dean Wang * and Zuolong Zhu
}

Department of Mechanical and Aerospace Engineering, The Ohio State University, $201 \mathrm{~W}$ 19th Avenue, Columbus, OH 43210, USA; zhu.2408@buckeyemail.osu.edu

* Correspondence: wang.12239@osu.edu

\section{check for}

updates

Citation: Wang, D.; Zhu, Z. A Revisit to CMFD Schemes: Fourier Analysis and Enhancement. Energies 2021, 14, 424. https://doi.org/10.3390/ en14020424

Received: 26 November 2020 Accepted: 10 January 2021 Published: 14 January 2021

Publisher's Note: MDPI stays neutral with regard to jurisdictional clai$\mathrm{ms}$ in published maps and institutional affiliations.

Copyright: (C) 2021 by the authors. Licensee MDPI, Basel, Switzerland. This article is an open access article distributed under the terms and conditions of the Creative Commons Attribution (CC BY) license (https:// creativecommons.org/licenses/by/ $4.0 /)$.

\begin{abstract}
The coarse-mesh finite difference (CMFD) scheme is a very effective nonlinear diffusion acceleration method for neutron transport calculations. CMFD can become unstable and fail to converge when the computational cell optical thickness is relatively large in k-eigenvalue problems or diffusive fixed-source problems. Some variants and fixups have been developed to enhance the stability of CMFD, including the partial current-based CMFD (pCMFD), optimally diffusive CMFD (odCMFD), and linear prolongation-based CMFD (lpCMFD). Linearized Fourier analysis has proven to be a very reliable and accurate tool to investigate the convergence rate and stability of such coupled high-order transport/low-order diffusion iterative schemes. It is shown in this paper that the use of different transport solvers in Fourier analysis may have some potential implications on the development of stabilizing techniques, which is exemplified by the odCMFD scheme. A modification to the artificial diffusion coefficients of odCMFD is proposed to improve its stability. In addition, two explicit expressions are presented to calculate local optimal successive overrelaxation (SOR) factors for lpCMFD to further enhance its acceleration performance for fixed-source problems and k-eigenvalue problems, respectively.
\end{abstract}

Keywords: neutron transport; Fourier analysis; SOR; CMFD; pCMFD; odCMFD; lpCMFD

\section{Introduction}

The coarse-mesh finite difference (CMFD) scheme, originally developed by Smith [1,2], is being widely used for accelerating neutron transport calculations. A well-known issue with CMFD is that the scheme can become unstable and even fail when the optical thickness becomes large, which has been reported in various numerical and theoretical studies. Some stabilization techniques have been developed to improve the stability of CMFD [3-6]. One simple method is the under-relaxation approach, which arbitrarily applies some damping on the drift flux coefficient or the scalar flux [4-6]. However, a judicious choice of under-relaxation has to be problem-dependent.

A variant of CMFD, called partial current-based CMFD (pCMFD), is found to be stable, but it is slower than the standard CMFD at an intermediate optical thickness [3]. Zhu et al. recently proposed an optimally diffusive CMFD (odCMFD) scheme, which generalizes pCMFD by adding some artificial diffusion to the diffusion coefficient of CMFD [7]. It is noted that caution should be exercised in determining adequate artificial diffusion. If the added diffusion is more than necessary, it would hurt the convergence rate; conversely, it would be insufficient for stability. In this paper, Fourier analysis and numerical results are presented to show that odCMFD can become unstable and even fail at a relatively large optical thickness because of insufficient diffusion. In this paper, it is shown that the use of different transport solvers in Fourier analysis can have some effect on the prediction of the convergence rate. A modification to the original artificial diffusion coefficients of odCMFD is proposed to improve its stability.

The linear prolongation-based CMFD (lpCMFD) scheme is the latest development in the CMFD family [8]. It replaces the conventional flat flux ratio-based scaling approach in the standard CMFD method with a linear interpolation of the scalar flux differences at the 
coarse-mesh cell edges between the high-order transport and low-order diffusion calculations. Various studies have demonstrated that lpCMFD has superior convergence performance in terms of stability and effectiveness, as compared to other CMFD schemes [9-14]. It is worth noting that lpCMFD does not use any "ad hoc" fixups, such as under-relaxation or artificial diffusion. Most recently, Wang has proposed to enhance the convergence performance of lpCMFD by updating the scalar flux (or the scattering source) with successive overrelaxation (SOR) $[15,16]$. It was found that lpCMFD with SOR can increase the convergence rate by almost $40 \%$ for neutron transport problems. However, the global optimal SOR value is problem-dependent. Instead of finding the global optimal SOR factor, the local SOR can be determined for each coarse-mesh cell based on its optical thickness and scattering ratio. In this paper, two explicit expressions are developed for the calculation of local optimal SOR factors-one is for fixed-source problems, and the other for k-eigenvalue problems, which can be easily implemented in a transport code.

The remainder of this paper is organized as follows: Section 2 presents the Fourier analysis of the CMFD schemes, such as CMFD, pCMFD, odCMFD, and lpCMFD, for both fixed-source problems and k-eigenvalue problems. The implications on the convergence rate of the step characteristic (SC) method and the diamond difference (DD) method for the transport solution are presented and discussed. In Section 3, the modification of odCMFD is discussed. Section 4 presents the two explicit expressions for the calculation of local SOR factors for lpCMFD. The numerical results of the one- and two-dimensional test problems are given in Section 5. Final conclusions are given in Section 6. A brief summary of the CMFD algorithm and formulation is presented in Appendix A.

\section{Fourier Analysis of CMFD Schemes}

The general algorithm and formulation of the coupled $S_{N}$ and CMFD iterative procedure is summarized in Appendix A. For simplicity, only the final Fourier analysis results of the CMFD schemes are presented for both fixed-source problems and k-eigenvalue problems. Detailed derivation and formulation can be found in the literature $[6-8,15,16]$.

\subsection{Spectral Radius for Fixed-Source and K-Eigenvalue Problems}

Fourier analysis is typically performed based on a 1D homogeneous problem with a uniform mesh and a specified number $\rho$ of fine-mesh cells per coarse cell. A single coarsemesh cell with periodic boundary conditions is employed. The coarse-mesh spacing is $\Delta$. The fine mesh spacing is $h=\frac{\Delta}{p}$. The monoenergetic $S_{N}$ transport equation is first linearized near the exact solution. Then, the error terms in the linearized equations are expressed as Fourier modes. The spectral radius can be obtained from the error iteration matrix.

For fixed-source problems, the final expression for the fine-mesh scalar flux iterative error modes is given as

$$
\boldsymbol{T}(\omega)=\boldsymbol{H}+\boldsymbol{\theta} \frac{\Delta\left(e^{i \Sigma_{t} \Delta \omega}-1\right) \boldsymbol{G}+\Sigma_{a} \Delta^{2} \boldsymbol{C}}{D\left[2 \cos \left(\Sigma_{t} \Delta \omega\right)-2\right]-\Sigma_{a} \Delta^{2}}
$$

where

$$
\begin{gathered}
\boldsymbol{H}=\sum_{n=1}^{N} w_{n} \boldsymbol{P}_{n} Y_{n}^{-1} \frac{\Sigma_{s}}{2} \\
\boldsymbol{C}=\frac{1}{p} \times\left[\begin{array}{ccc}
1 & \cdots & 1
\end{array}\right]_{1 \times p} \boldsymbol{H} \\
\boldsymbol{P}_{n}=\left[\begin{array}{ccccc}
\boldsymbol{G}=\left[\begin{array}{ccccc}
1 & 0 & \cdots & 0
\end{array}\right]_{1 \times p} \boldsymbol{H} \\
\frac{1-\alpha_{n}}{2} & \frac{1+\alpha_{n}}{2} & 0 & 0 & 0 \\
0 & \frac{1-\alpha_{n}}{2} & \frac{1+\alpha_{n}}{2} & 0 & 0 \\
0 & 0 & 0 & \ddots & 0 \\
\frac{1+\alpha_{n}}{2} e^{i \Sigma_{t} \Delta \omega} & 0 & 0 & 0 & \frac{1-\alpha_{n}}{2}
\end{array}\right]_{p \times p}
\end{gathered}
$$




$$
\begin{gathered}
Y_{n}=\left[\begin{array}{ccccc}
a & b & 0 & 0 & 0 \\
0 & a & b & 0 & 0 \\
0 & 0 & 0 & \ddots & 0 \\
b e^{i \Sigma_{t} \Delta \omega} & 0 & 0 & 0 & a
\end{array}\right]_{p \times p} \\
a=-\frac{\mu_{n}}{h}+\Sigma_{t}\left(\frac{1-\alpha_{n}}{2}\right) \\
\alpha_{n}=\left\{\begin{array}{c}
\frac{1+e^{-\frac{\Sigma_{t} h}{\mu_{h}}}-\frac{2 \mu_{n}}{\Sigma_{t} h}}{1-e^{-\frac{\Sigma_{t} h}{\mu_{h}}}} \text { for SC } \\
0 \\
h=\frac{\Delta}{p} \\
D=\frac{1}{3 \Sigma_{t}}
\end{array}\right.
\end{gathered}
$$

Note that $\mu_{n}$ and $w_{n}$ are the nodes and weights of a quadrature set (e.g., GaussLegendre quadrature).

The vector $\boldsymbol{\theta}$ is defined as

$$
\boldsymbol{\theta}=\left(\theta_{1} \theta_{2} \cdots \theta_{p}\right)^{T}
$$

The following different iterative schemes can be obtained by defining the vector $\boldsymbol{\theta}$ as

$$
\begin{gathered}
\text { SI }: \theta_{k}=0 . \\
\text { CMFD : } \theta_{k}=1 . \\
\text { pCMFD : } \theta_{k}=\frac{1}{1+\frac{3}{4} \tau} . \\
\text { odCMFD : } \theta_{k}=\theta(\tau) . \\
\text { lpCMFD : } \theta_{k}=\frac{2 p+(2 p-2 k+1) e^{-i \tau \omega}+(2 k-1) e^{i \tau \omega}}{4 p} .
\end{gathered}
$$

where $1 \leq k \leq p$, and the coarse-mesh optical thickness $\tau=\Sigma_{t} \Delta$.

It can be seen that when $\theta_{k}=0$, it reverts to the transport source iteration (SI) method with no CMFD acceleration. For the standard CMFD scheme, $\theta_{k}=1$, which means a flat prolongation approach is used. The function of $\theta_{k}$ for pCMFD was derived by Jarret et al. [6], and it implies that pCMFD tends to the CMFD scheme when the coarse-mesh cell optical thickness $\tau\left(=\Sigma_{t} \Delta\right)$ becomes large. The $\theta_{k}$ of odCMFD is a function of $\tau$, described by Zhu et al. [7]. The $\theta_{k}$ values of lpCMFD are reported by Wang and Xiao [8].

The spectral radius of the CMFD schemes as a function of Fourier frequency $\omega$ is given as

$$
\rho(\omega)=\max (\operatorname{abs}(e i g(\boldsymbol{T}(\omega)))) .
$$

where Fourier frequency is expressed as follows

$$
\begin{gathered}
\omega=\frac{2 \pi s}{\sum_{t} L}, s=1,2, \ldots, J, \\
J=\frac{L}{\Delta} .
\end{gathered}
$$

$L$ is the domain size. Finally, the spectral radius is defined as

$$
\rho=\max (\rho(\omega))
$$


For k-eigenvalue problems, the final expression for the fine-mesh scalar flux iterative error modes is given as

$$
\boldsymbol{T}(\omega)=\boldsymbol{H}+\boldsymbol{\theta} \frac{\Delta\left(e^{i \Sigma_{t} \Delta \omega}-1\right) \boldsymbol{G}}{D\left[2 \cos \left(\Sigma_{t} \Delta \omega\right)-2\right]}
$$

where

$$
\begin{gathered}
\boldsymbol{H}=\sum_{n=1}^{N} w_{n} \boldsymbol{P}_{n} Y_{n}^{-1} \frac{\Sigma_{t}}{2}, \\
\boldsymbol{G}=\left[\begin{array}{ccccc}
1 & 0 & \cdots & 0
\end{array}\right]_{1 \times p} \boldsymbol{H} .
\end{gathered}
$$

Compared to the expression for the fixed-source case, only matrices $\boldsymbol{H}$ and $\boldsymbol{G}$ are different, in which the scattering cross-section is replaced with the total cross-section. Therefore, the spectral radius is independent of scattering for k-eigenvalue problems. It is interesting to note that the spectral radius of fixed-source problems will be limited to that of k-eigenvalue problems, as the scattering ratio $c \rightarrow 0$ (pure scattering).

\subsection{The Implication of Transport Solver}

In Fourier analysis, it is customary to use the SC method to discretize the $S_{N}$ transport equation, in which the transport angular flux on each computational cell is weighted as

$$
\psi_{n, k}=\left(\frac{1-\alpha_{n}}{2}\right) \psi_{n, k-\frac{1}{2}}+\left(\frac{1+\alpha_{n}}{2}\right) \psi_{n, k+\frac{1}{2}}
$$

where $1 \leq k \leq p \cdot \psi_{n, k-\frac{1}{2}}$ and $\psi_{n, k+\frac{1}{2}}$ are the left and right edge fluxes of the $k-t h$ cell. Then,

$$
\alpha_{n}=\frac{1+e^{-\frac{\Sigma_{t} h}{\mu_{n}}}}{1-e^{-\frac{\Sigma_{t} h}{\mu_{n}}}}-\frac{2 \mu_{n}}{\Sigma_{t} h} .
$$

Remark 1. If the cell optical thickness $\Sigma_{t} h \rightarrow 0$, then $\alpha_{n} \rightarrow 0$, thus the SC solution tends to the $D D$ solution; if $\Sigma_{t} h \rightarrow \infty$, then it tends to the first-order upwind method (i.e., the step difference method) because of $\alpha_{n} \rightarrow 1$ for $\mu_{n}>0$, and $\alpha_{n} \rightarrow-1$ for $\mu_{n}<0$. Therefore, there should be no difference in the spectral radius predicted by Fourier analysis when using the SC or the DD method at a thin optical thickness. However, the difference will become appreciable when the coarse-mesh optical thickness $\tau>1$. This is confirmed by the Fourier analysis as shown in Figure 1. Note that the results are obtained with the domain size of $L=200 \mathrm{~cm}$, the coarse-mesh size $\Delta=1 \mathrm{~cm}$, and the number of fine cells in each coarse cell $p=10$.

The following observations can be drawn from the above Fourier analyses:

1. For CMFD, the results of the SC and DD are very close (the black and blue curves are almost coincident), and both solvers predict failure when $\tau>1$.

2. For $\mathrm{pCMFD}$, the results of the SC and DD agree very well within the differences of less than 0.006, except that the DD predicts failure when $\tau>90$.

3. For odCMFD, significant differences show up when $\tau>2.5$. The DD predicts failure at $\tau>5.5$, while the SC results show that the scheme is more stable and becomes unstable until $\tau>20$. It is worth mentioning that the SC solution is somewhat sensitive to the domain size. If a smaller domain is used, e.g., $L=100 \mathrm{~cm}$, then there is no occurrence of a failure in the SC solution.

4. For lpCMFD, the SC results show that the scheme becomes unstable when $\tau>27$, but interestingly the DD does not predict any failure. Numerical testing indicates that the lpCMFD scheme is stable even up to a much larger optical thickness, which implies that the SC prediction is less reliable. 


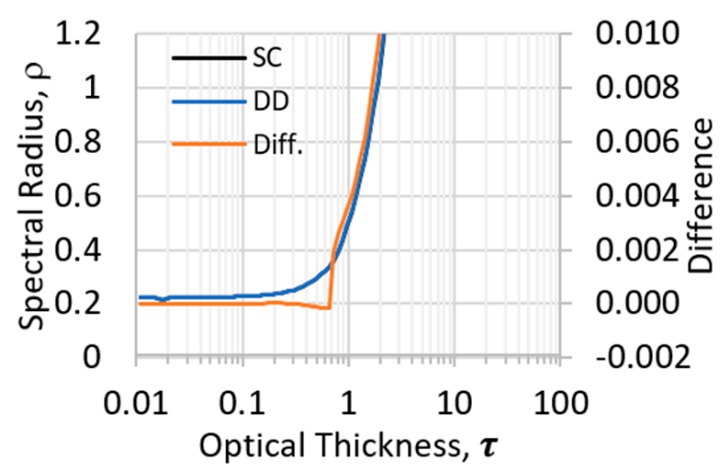

(a)

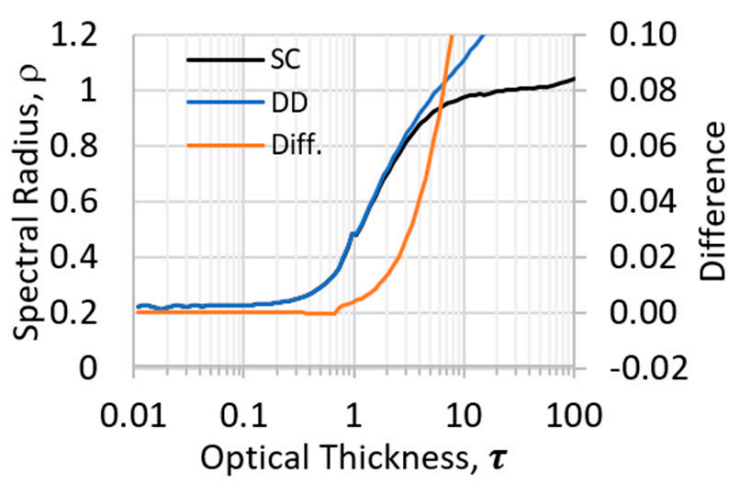

(c)

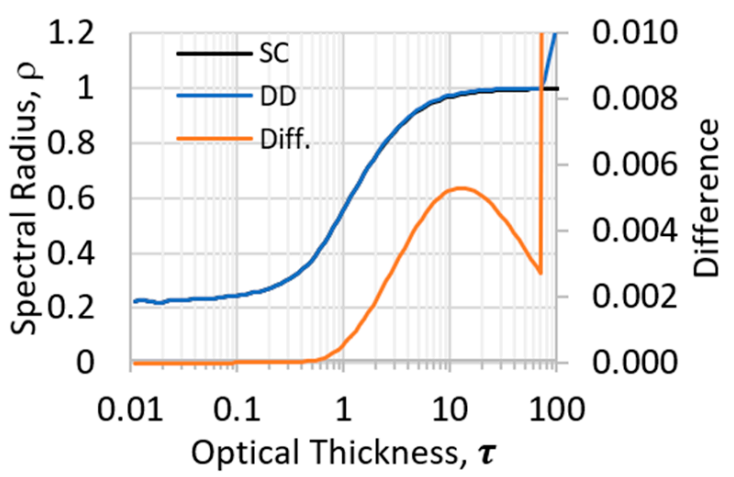

(b)

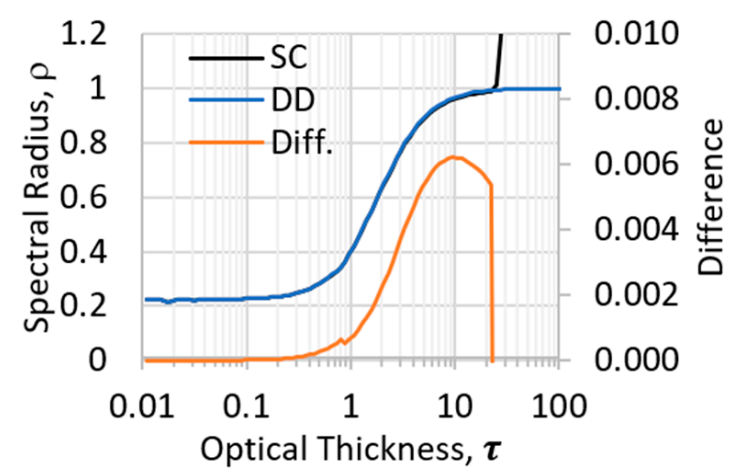

(d)

Figure 1. Spectral radius vs. optical thickness for the k-eigenvalue case. (a) Coarse-mesh finite difference (CMFD); (b) partial current-based CMFD (pCMFD); (c) optimally diffusive CMFD (odCMFD); (d) linear prolongation-based CMFD (lpCMFD).

Now that the spectral radius of the DD is slightly larger than that of the SC, the question arises: which transport solver (DD or SC) should be used in Fourier analysis? It is less of a problem for CMFD, pCMFD, and lpCMFD, since both transport solvers agree very well, except for very large optical thicknesses. However, this is not the case for the odCMFD, which shows that the DD results are quite different from those of the SC. The reason is that the original optimal artificial diffusion coefficients were determined based on the SC solution, and they are slightly insufficient for the DD solution. In the next section, it will be shown that the scheme can be stabilized by using the DD-based results.

In addition, it should be noted that when the optical thickness becomes large, the spectral radius gradually reaches the spectral radius of the power (or source) iteration scheme, $\rho=\frac{\tan ^{-1}\left(\frac{\pi}{\Sigma_{t} L}\right)}{\frac{\pi}{\Sigma_{t} L}}$, which becomes almost 1 for the relatively large domain. Therefore, the convergence or stability of CMFD at a large optical thickness has the least practical importance, in particular for neutron transport problems.

\section{Modification of odCMFD}

Zhu et al. optimized the artificial diffusion coefficients based on Fourier analysis with the SC for the transport solution [7]. The odCMFD generalizes the CMFD by defining the modified diffusion coefficient as

$$
\widetilde{D}=\frac{1}{3 \Sigma_{t, C M}}+\theta \Delta
$$


where the artificial diffusion coefficient is defined as

$$
\theta= \begin{cases}0, & \tau<1 \\ \sum_{i=0}^{6} a_{i} \tau^{i}, & 1 \leq \tau<14, \\ 0.127, & \tau \geq 14\end{cases}
$$

which is a function of the coarse-mesh cell optical thickness $\tau=\Sigma_{t, C M} \Delta$. The polynomial coefficients $a_{i}$ are given in Table 1 [7]. The Fourier analysis in the previous section shows that odCMFD can lose its effectiveness and even become unstable at a relatively large optical thickness. The following modified artificial diffusion coefficients are obtained by using the DD instead of the SC in the Fourier analysis.

$$
\theta_{\text {mod }}=\left\{\begin{array}{ll}
0, & \tau<0.8 \\
\sum_{i=0}^{6} a_{i} \tau^{i}, & 0.8 \leq \tau<14 \\
0.1685, & 14 \leq \tau<28 \\
0.1685+0.002067(\tau-28), & 28 \leq \tau<140 \\
>0.4, & \tau>140
\end{array},\right.
$$

where the modified coefficients $a_{i}$ are given in Table 1. A polynomial function is used for $0.8 \leq \tau<14$, which is of practical importance for neutron transport problems. A simple linear function is used for $28 \leq \tau<140$, and a larger value is suggested for $\tau>140$.

Table 1. Coefficients for the polynomial fitting of optimal $\theta$.

\begin{tabular}{ccc}
\hline $\boldsymbol{i}$ & Original $\boldsymbol{a}_{\boldsymbol{i}}[7]$ & Modified $\boldsymbol{a}_{\boldsymbol{i}}$ \\
\hline 0 & $-5.543 \times 10^{-2}$ & $-1.764 \times 10^{-2}$ \\
1 & $8.741 \times 10^{-2}$ & $7.156 \times 10^{-2}$ \\
2 & $-2.153 \times 10^{-2}$ & $-1.409 \times 10^{-2}$ \\
3 & $3.146 \times 10^{-3}$ & $1.591 \times 10^{-3}$ \\
4 & $-2.684 \times 10^{-4}$ & $-1.012 \times 10^{-4}$ \\
5 & $1.223 \times 10^{-5}$ & $3.372 \times 10^{-6}$ \\
6 & $-2.285 \times 10^{-7}$ & $-4.557 \times 10^{-8}$ \\
\hline
\end{tabular}

Figure 2a shows that the modified optimal $\theta$ values are larger than the original ones, implying that more artificial diffusion should be added to the CMFD scheme. Herein, the odCMFD scheme with the new $\theta$ function is called "modCMFD". Figure $2 b$ illustrates the spectral radius curves of modCMFD for the SC and DD, respectively. It can be seen that both transport solvers predict almost the same convergence rate with the modified artificial diffusion coefficients, and the scheme is stable for any optical thickness.

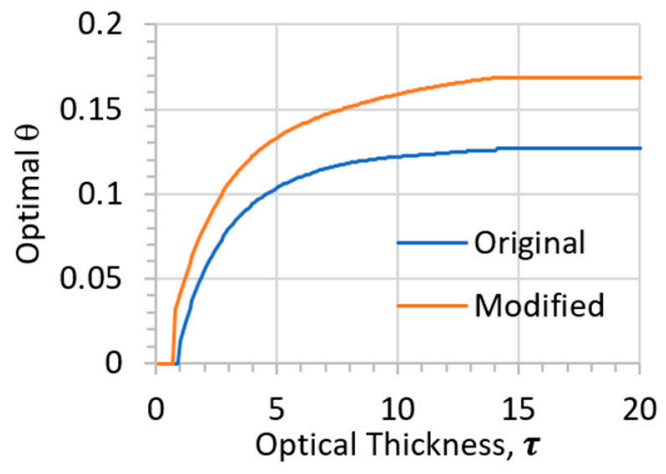

(a)

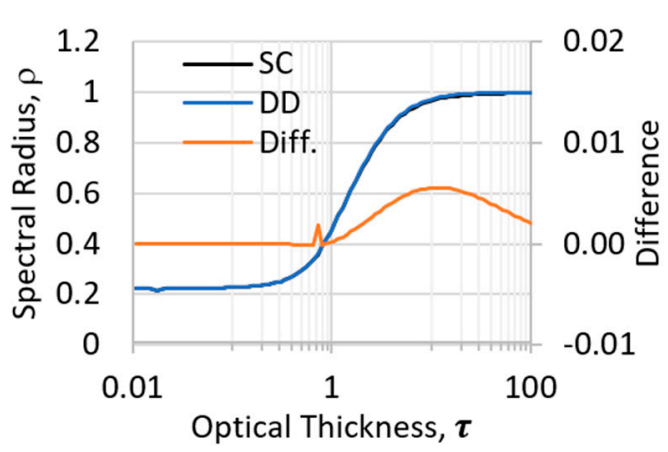

(b)

Figure 2. Results for the k-eigenvalue case. (a) Modified $\theta$; (b) Spectral radius of odCMFD scheme with the new $\theta$ function (modCMFD). 


\section{Optimal SOR for lpCMFD}

It has been illustrated that the lpCMFD can be enhanced with SOR. For the fixedsource case, the Fourier analysis indicates that the optimal SOR factor depends on not only the optical thickness, but also the scattering ratio [15], while for the k-eigenvalue case, it only depends on the optical thickness [16]. However, it is found in numerical testing that for better performance, some damping (relaxation) may be necessary for the SOR factors predicted by the Fourier analysis, in particular, for large scattering ratios. In this section, two explicit expressions are developed to analytically determine the local optimal SOR factors for fixed-source problems and k-eigenvalue problems, respectively.

\subsection{SOR for Fixed-Source Problems}

The spectral radius of the lpCMFD scheme for fixed-source problems is a function of the coarse-mesh cell optical thickness and scattering ratio. Therefore, the optimal SOR factor should also depend on optical thickness and scattering.

Based on a comprehensive Fourier analysis, optimal SOR factors are obtained for the optical thickness in the range of $0.1 \leq \tau \leq 20$, and the scattering ratio in the range of $0.1 \leq c \leq 0.998$. The SOR distribution in terms of the optical thickness and scattering ratio is plotted in Figure 3.

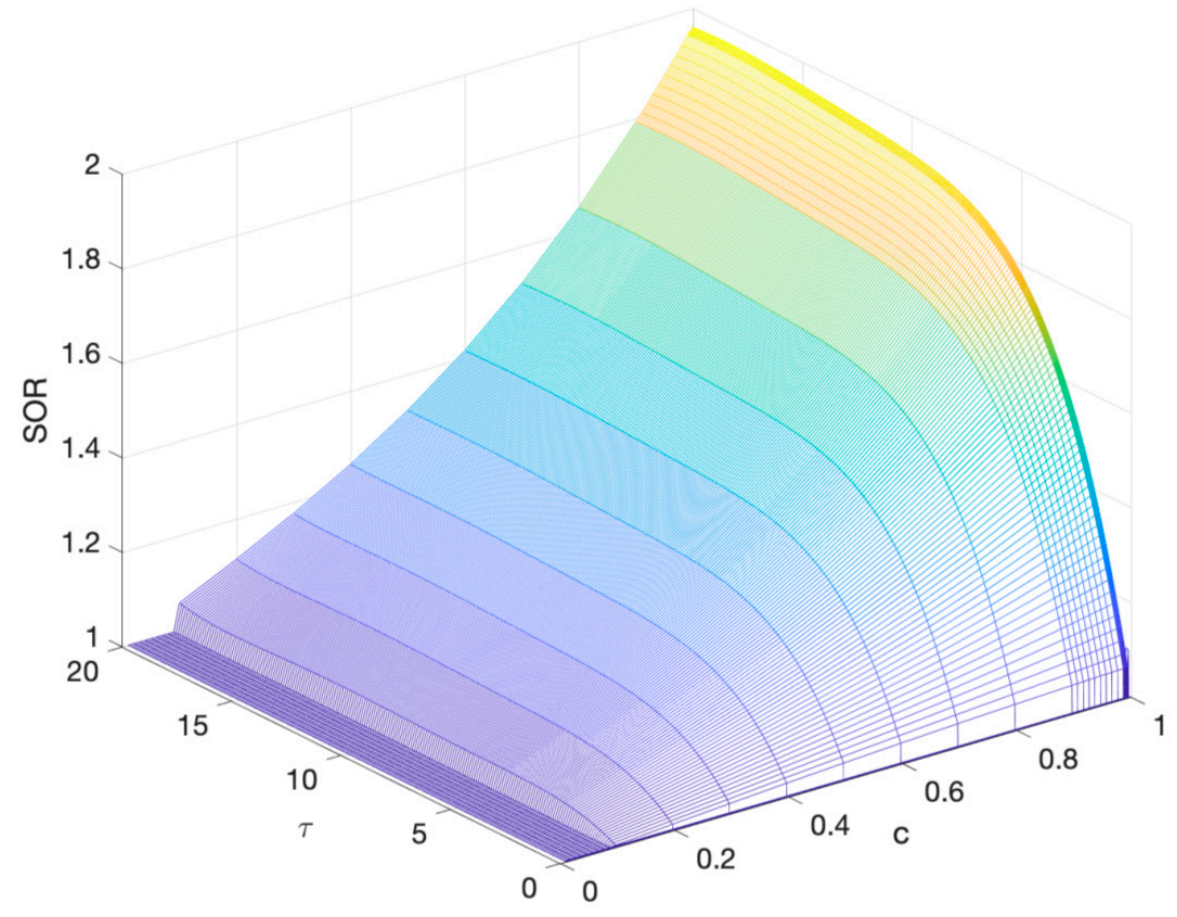

Figure 3. Successive overrelaxation (SOR) distribution.

The fitted SOR function is given as

$$
\mathrm{SOR}_{\mathrm{FA}}=\sum_{i=0}^{5} \sum_{j=0}^{3} a_{i, j} \tau^{i} c^{j}, \quad i+j \leq 5
$$

Note that $\mathrm{SOR}=1$ when $c<0.1, \mathrm{SOR}=1.1$ when $c<0.998$, and $\mathrm{SOR}=\mathrm{SOR}(\tau=20)$ for $\tau<20$. The polynomial fitting coefficients are given in Table 2 . 
Table 2. Polynomial fitting coefficients.

\begin{tabular}{cc}
\hline Order $(i, j)$ & Coefficient $\left(a_{i, j}\right)$ \\
\hline$(0,0)$ & $9.898 \times 10^{-1}$ \\
$(1,0)$ & $1.241 \times 10^{-2}$ \\
$(0,1)$ & $8.727 \times 10^{-2}$ \\
$(2,0)$ & $-4.468 \times 10^{-3}$ \\
$(1,1)$ & $1.579 \times 10^{-1}$ \\
$(0,2)$ & $-3.770 \times 10^{-2}$ \\
$(3,0)$ & $6.021 \times 10^{-4}$ \\
$(2,1)$ & $-2.131 \times 10^{-2}$ \\
$(1,2)$ & $4.672 \times 10^{-2}$ \\
$(0,3)$ & $-4.223 \times 10^{-3}$ \\
$(4,0)$ & $-3.473 \times 10^{-5}$ \\
$(3,1)$ & $1.316 \times 10^{-3}$ \\
$(2,2)$ & $-7.051 \times 10^{-3}$ \\
$(1,3)$ & $6.083 \times 10^{-2}$ \\
$(5,0)$ & $7.160 \times 10^{-7}$ \\
$(4,1)$ & $-2.931 \times 10^{-5}$ \\
$(3,2)$ & $2.328 \times 10^{-4}$ \\
$(2,3)$ & $-1.871 \times 10^{-3}$ \\
\hline
\end{tabular}

It is seen that SOR increases with the optical thickness and scattering ratio. In practical implementation, some relaxation is needed for relatively large scattering ratios because of numerical effects, such as the treatment of boundary conditions. To apply the relaxation, the domain of the scattering ratio $(c \in[0.1,0.998])$ is divided into three regions:

- $\quad$ Region 1: $0.1 \leq c<0.9$;

- Region 2: $0.9 \leq c<0.99$;

- Region 3: $0.99 \leq c \leq 0.998$.

The numerical results indicate that no relaxation is needed for Region 1 . However, for both Regions 2 and 3, the relaxation is required. In order to obtain better fitting, the following scaling is used for Regions 2 and 3.

$$
\left\{\begin{array}{ll}
c_{\text {scaled }}=\frac{9 *(c-0.9)}{0.098}+1, & 0.9 \leq c<0.99 \\
c_{\text {scaled }}=\frac{9 *(c-0.99)}{0.008}+1, & 0.99<c \leq 0.998
\end{array} .\right.
$$

The relaxation functions for Regions 2 and 3 are given as

$$
\begin{aligned}
& \text { Relax1 }=\sum_{i=0}^{4} \sum_{j=0}^{5} b_{i, j} \tau^{i} c_{\text {scaled }}^{j}, \quad i+j \leq 5, \quad(0.9 \leq c<0.99), \\
& \text { Relax2 }=\sum_{i=0}^{4} \sum_{j=0}^{5} c_{i, j} \tau^{i} c_{\text {scaled }}^{j}, \quad i+j \leq 5, \quad(0.99 \leq c<0.998) .
\end{aligned}
$$

The polynomial fitting coefficients $b_{i, j}$ and $c_{i, j}$ are given in Table 3 .

The final function of SOR is given as

$$
\mathrm{SOR}=\left\{\begin{array}{ll}
\mathrm{SOR}_{\mathrm{FA}}, & c<0.9 \\
\mathrm{SOR}_{\mathrm{FA}} * \text { Relax1, } & 0.9 \leq c<0.99 \\
\mathrm{SOR}_{\mathrm{FA}} * \text { Relax2, } & c \geq 0.99
\end{array} .\right.
$$

The relaxed SOR distribution in terms of the optical thickness and scattering ratio is plotted in Figure 4. 
Table 3. Polynomial fitting coefficients for relaxation factors.

\begin{tabular}{ccc}
\hline Order $(i, j)$ & Coefficient $\left(\boldsymbol{b}_{i, j}\right)$ & Coefficient $\left(c_{i, j}\right)$ \\
\hline$(0,0)$ & $1.025 \times 10^{+0}$ & $9.851 \times 10^{-1}$ \\
$(1,0)$ & $-5.911 \times 10^{-3}$ & $-1.978 \times 10^{-2}$ \\
$(0,1)$ & $-1.027 \times 10^{-3}$ & $-1.975 \times 10^{-2}$ \\
$(2,0)$ & $8.845 \times 10^{-4}$ & $1.988 \times 10^{-3}$ \\
$(1,1)$ & $7.404 \times 10^{-4}$ & $-5.955 \times 10^{-3}$ \\
$(0,2)$ & $5.243 \times 10^{-2}$ & $1.422 \times 10^{-2}$ \\
$(3,0)$ & $-7.166 \times 10^{-5}$ & $-6.609 \times 10^{-5}$ \\
$(2,1)$ & $-2.291 \times 10^{-4}$ & $3.229 \times 10^{-4}$ \\
$(1,2)$ & $-2.535 \times 10^{-3}$ & $7.285 \times 10^{-4}$ \\
$(0,3)$ & $-1.216 \times 10^{-2}$ & $-3.725 \times 10^{-3}$ \\
$(4,0)$ & $1.562 \times 10^{-6}$ & $8.453 \times 10^{-7}$ \\
$(3,1)$ & $6.605 \times 10^{-6}$ & $-6.618 \times 10^{-7}$ \\
$(2,2)$ & $2.695 \times 10^{-5}$ & $-3.883 \times 10^{-5}$ \\
$(1,3)$ & $3.953 \times 10^{-4}$ & $-4.949 \times 10^{-5}$ \\
$(0,4)$ & $1.251 \times 10^{-3}$ & $3.988 \times 10^{-4}$ \\
$(4,1)$ & $7.090 \times 10^{-8}$ & $-2.618 \times 10^{-7}$ \\
$(3,2)$ & $-1.480 \times 10^{-6}$ & $9.277 \times 10^{-7}$ \\
$(2,3)$ & $1.859 \times 10^{-6}$ & $8.170 \times 10^{-7}$ \\
$(1,4)$ & $-2.373 \times 10^{-5}$ & $5.419 \times 10^{-7}$ \\
$(0,5)$ & $-4.642 \times 10^{-5}$ & $-1.515 \times 10^{-5}$ \\
\hline
\end{tabular}

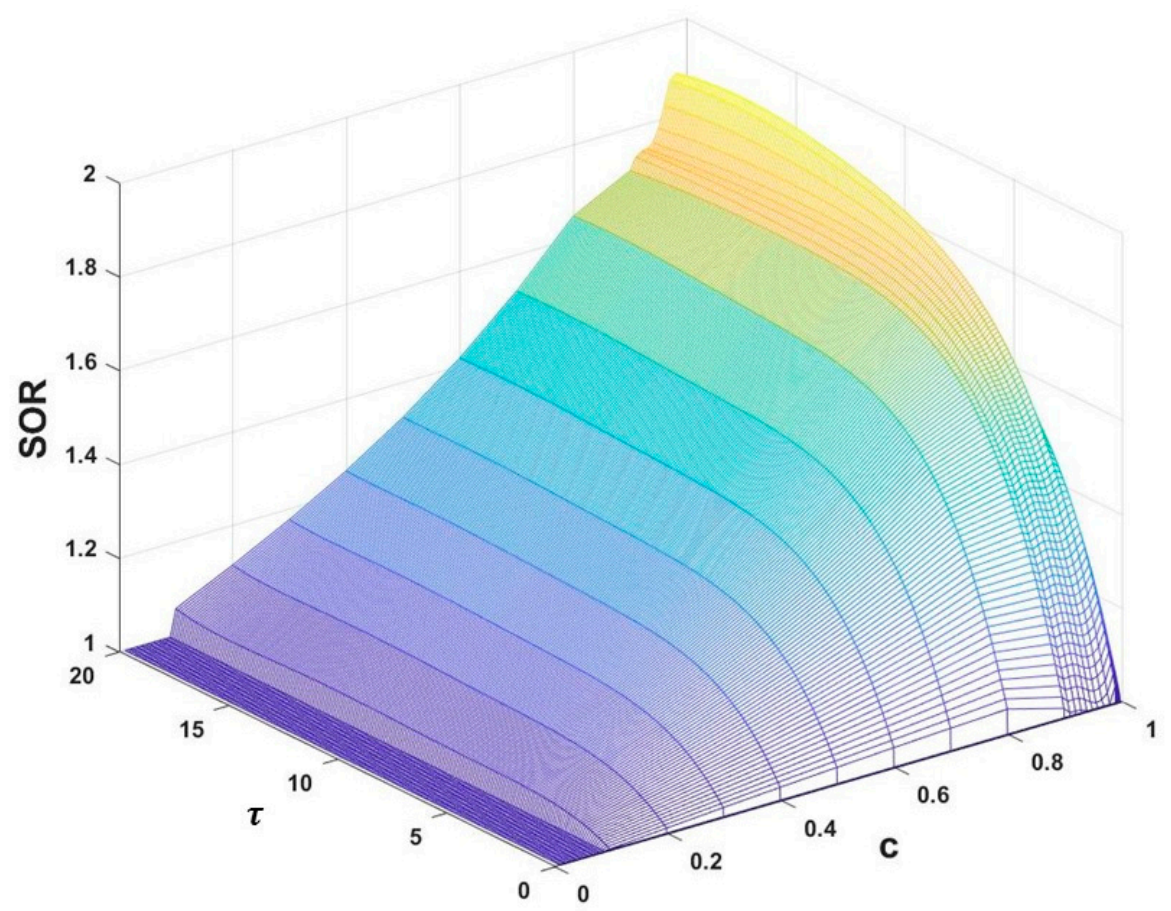

Figure 4. Modified SOR distribution.

\subsection{SOR for K-Eigenvalue Problems}

As discussed in Section 2.1, differing from fixed-source problems, the spectral radius of the CMFD schemes for k-eigenvalue problems is dependent on the coarse-mesh optical thickness, but independent of scattering. 
Based on the Fourier analysis, optimal SOR factors are found for the optical thickness in the range of $0.1 \leq \Sigma_{t, C M} \Delta \leq 20$. The fitted SOR function is given as

$$
\mathrm{SOR}_{\mathrm{FA}}=\sum_{i=0}^{6} a_{i} \tau^{i}, \quad 0.1 \leq \tau \leq 20 .
$$

When $\tau<0.1$, SOR $=1$, and when $\tau>20$, SOR $=\operatorname{SOR}(\tau=20)$. The polynomial fitting coefficients are given in Table 4, and the function above is plotted in Figure 5.

Table 4. Polynomial fitting coefficients.

\begin{tabular}{cc}
\hline Order $(i)$ & Coefficient $\left(a_{i}\right)$ \\
\hline 0 & $1.019 \times 10^{+0}$ \\
1 & $2.474 \times 10^{-1}$ \\
2 & $-2.030 \times 10^{-2}$ \\
3 & $-8.307 \times 10^{-4}$ \\
4 & $2.174 \times 10^{-4}$ \\
5 & $-1.163 \times 10^{-5}$ \\
6 & $2.049 \times 10^{-7}$ \\
\hline
\end{tabular}

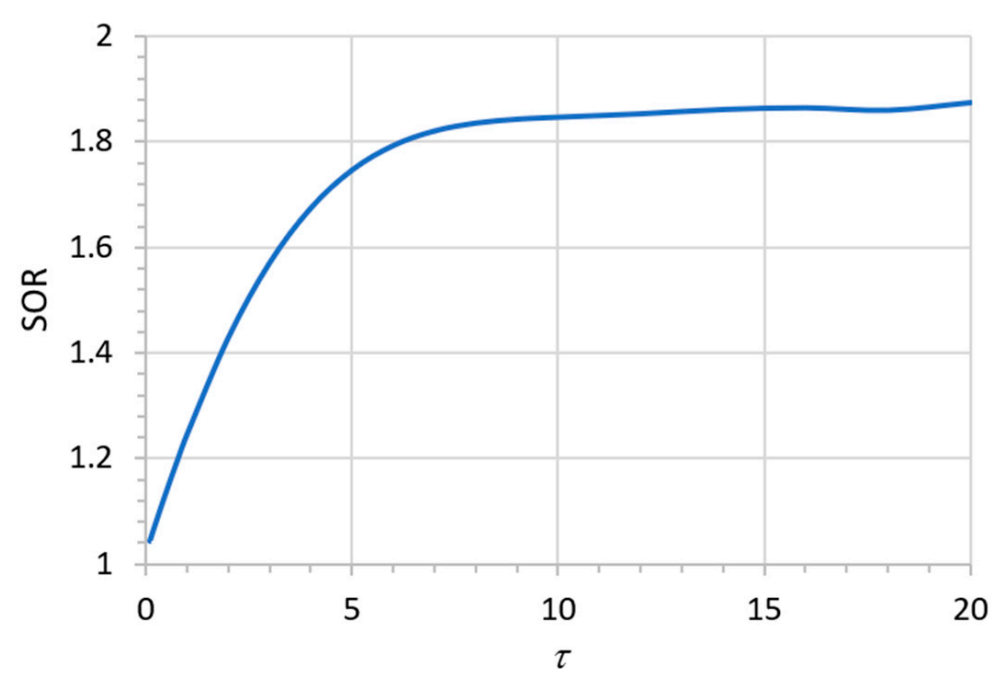

Figure 5. SOR vs. $\tau$.

This shows that for k-eigenvalue problems, the SOR factor increases with $\tau$ significantly when $\tau<8$, and after that, the SOR is almost a constant. However, for numerical implementation, some relaxation should be applied to the SOR factor. A relaxation factor of 0.9 is recommended for the entire domain, and additional relaxation is needed for high scattering ratios.

The final expression of SOR for k-eigenvalue problems is summarized as follows:

$$
\mathrm{SOR}=0.9 * \begin{cases}\mathrm{SOR}_{\mathrm{FA}}, & c<0.8 \\ \mathrm{SOR}_{\mathrm{FA}} *\left[1+(\operatorname{Relax}-1) \frac{c-0.8}{0.19}\right], & 0.8<c<0.99 \\ \mathrm{SOR}_{\mathrm{FA}} * \text { Relax }, & c \geq 0.99\end{cases}
$$

where

$$
\text { Relax }=\left\{\begin{array}{ll}
1, & \tau<4.35 \\
1.1 \tau-0.0237, & \tau \geq 4.35
\end{array} .\right.
$$

It should be noted that if the relaxed SOR is smaller than 1 , then $\mathrm{SOR}=1$. 


\section{Numerical Results}

The numerical results of the one- and two-dimensional test problems are presented to demonstrate the convergence performance of the modCMFD and the lpCMFD with SOR (lpCMFD-SOR). For comparison, the results of CMFD and pCMFD are presented as well.

\subsection{One-Dimensional Iron-Water Test Problem}

The 1D modified iron-water test problem is defined in Figure $6[9,12,15]$. The problem domain is uniformly divided into 30 coarse-mesh cells with a mesh size of $\Delta=1.0 \mathrm{~cm}$, and each coarse-mesh cell consists of 10 uniform fine-mesh cells. The high-order $S_{N}$ neutron transport equation is discretized by using the Gauss-Legendre $S_{12}$ quadrature set for the angle, and the SC and DD methods for space, respectively. The $S_{N}$ equation is solved on the fine-mesh grid. The low-order CMFD equation is discretized and solved on the coarse-mesh grid to accelerate the $S_{N}$ source iteration. The numerical results are shown in Figure 7.

This shows that the source iteration (SI) scheme converges very slowly because the problem is relatively thick and diffusive $\left(\tau=\Sigma_{t, C M} \Delta=3.3333\right.$ and $\left.c=0.9941\right)$. The CMFD fails to converge. Note that the original odCMFD scheme is slower than the pCMFD for this problem. The modCMFD can improve the convergence rate significantly, and is faster than the pCMFD, as supposed. It can be seen that the original odCMFD is sensitive to the $S_{N}$ transport solver. It converges more slowly for the DD than SC, which is consistent with the theoretical finding. The modCMFD is not sensitive to the transport solver and it yields almost the same performance for both the DD and SC. As expected, the lpCMFD scheme performs best among all the CMFD schemes. More interestingly, the lpCMFD-SOR scheme can further accelerate the convergence, in which the local optimal SOR factor for each coarse-mesh cell is precomputed by Equation (34). The results are similar to the case reported by Wang [15], where a global SOR is used.

\begin{tabular}{|c|c|c|c|c|c|}
\hline 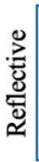 & $\begin{array}{c}\begin{array}{c}\text { Region 1 } \\
\text { Water }\end{array} \\
\mathrm{Q}=1.0 \\
\Sigma_{t}=3.3333 \\
\Sigma_{s}=3.3136\end{array}$ & 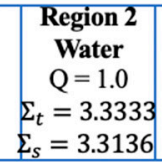 & $\begin{array}{c}\begin{array}{c}\text { Region 3 } \\
\text { Iron }\end{array} \\
\mathrm{Q}=1.0 \\
\Sigma_{t}=1.3333 \\
\Sigma_{s}=1.1077\end{array}$ & 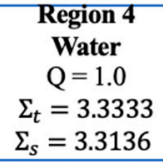 & 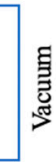 \\
\hline & & 12 & & & $0 \mathrm{~cm}$ \\
\hline
\end{tabular}

Figure 6. 1D iron-water problem specifications.

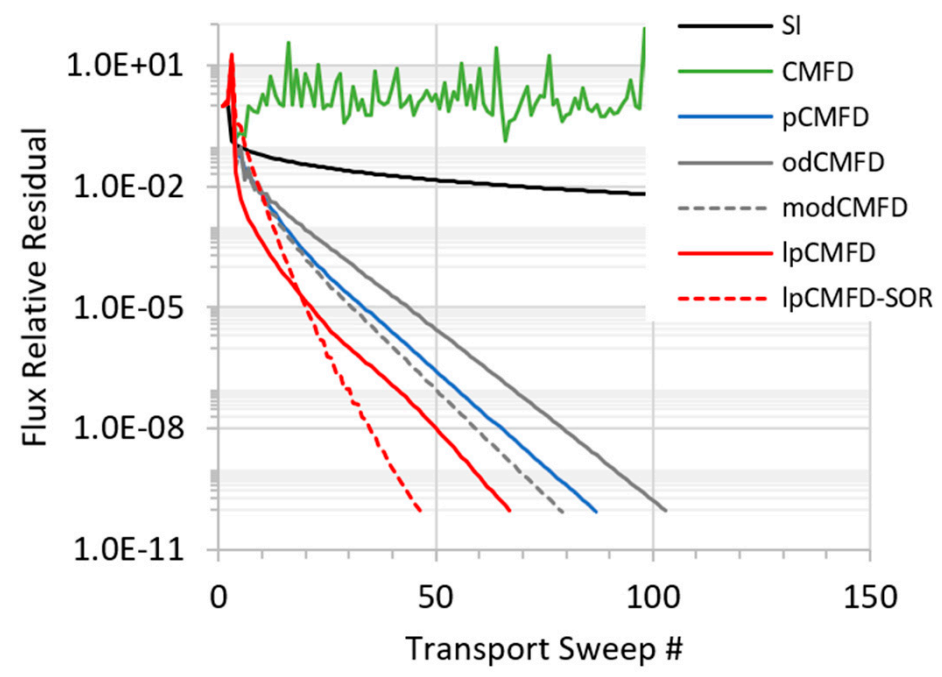

(a)

Figure 7. Cont. 


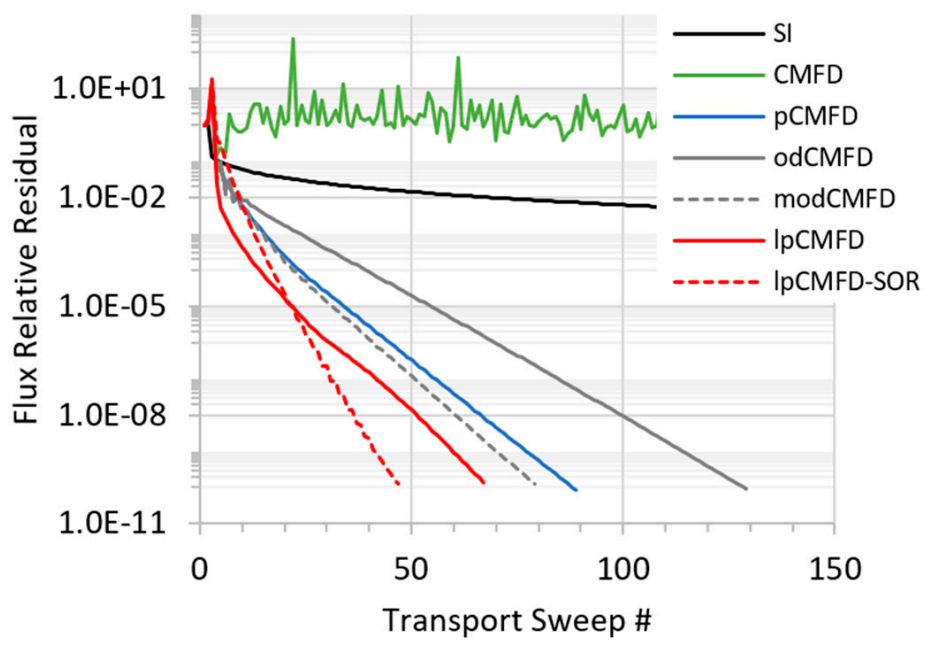

(b)

Figure 7. Results of the 1D iron-water problem. (a) Convergence, step characteristic (SC); (b) Convergence, diamond difference (DD).

\subsection{Two-Dimensional Test Problem}

The second test case is a 2D k-eigenvalue problem as shown in Figure 8. The problem domain is a $6 \times 6 \mathrm{~cm}$ square with the reflective boundary condition on each side. The domain is divided into $5 \times 5$ uniform coarse-mesh cells, with each consisting of $10 \times 10$ fine-mesh cells. The numerical solutions for the $S_{N}$ transport are obtained on the fine-mesh $(50 \times 50)$ using the level-symmetric $S_{12}$ quadrature set for angular discretization and the DD method for spatial discretization. The CMFD solutions are obtained on the coarse-mesh grids $(5 \times 5)$. Two cases are presented for the problem: Case A has the scattering ratio of 0.9, and it is 0.7 in Case $B$. The problem is to investigate whether the scattering has any impact on the convergence, although the Fourier analysis shows that the spectral radius of the CMFD schemes should be independent of scattering.

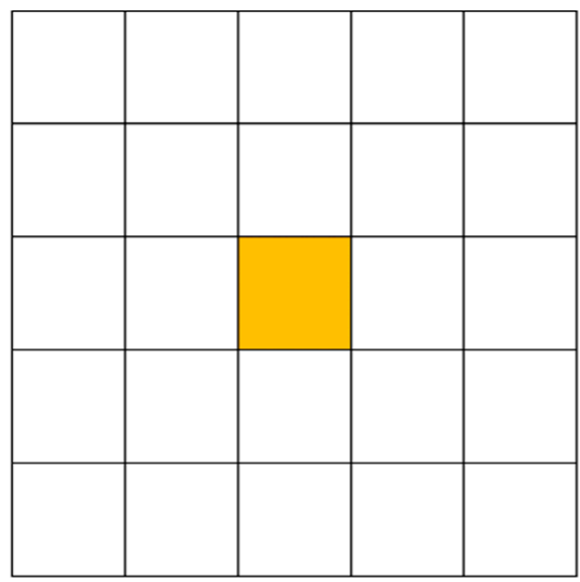

$$
\begin{aligned}
& \Sigma_{t}=5 \mathrm{~cm}^{-1}, c=0.9\left(\text { Case A) and } 0.7 \text { (Case B), } v \Sigma_{f}=0.5 \mathrm{~cm}^{-1}\right. \\
& \Sigma_{t}=5 \mathrm{~cm}^{-1}, c=0.9 \text { (Case A) and } 0.7 \text { (Case B), } v \Sigma_{f}=0.51 \mathrm{~cm}^{-1}
\end{aligned}
$$

Figure 8. 2D k-eigenvalue test problem. 
The convergence performance is illustrated in Figure 9. It is interesting to notice that the scattering does have some effect on the convergence of the pCMFD and odCMFD. In general, the convergence slows down as the scattering ratio decreases. However, both the modCMFD and lpCMFD are much less sensitive to the scattering. Note that the local SOR factors of the coarse-mesh cells are calculated according to Equation (36).

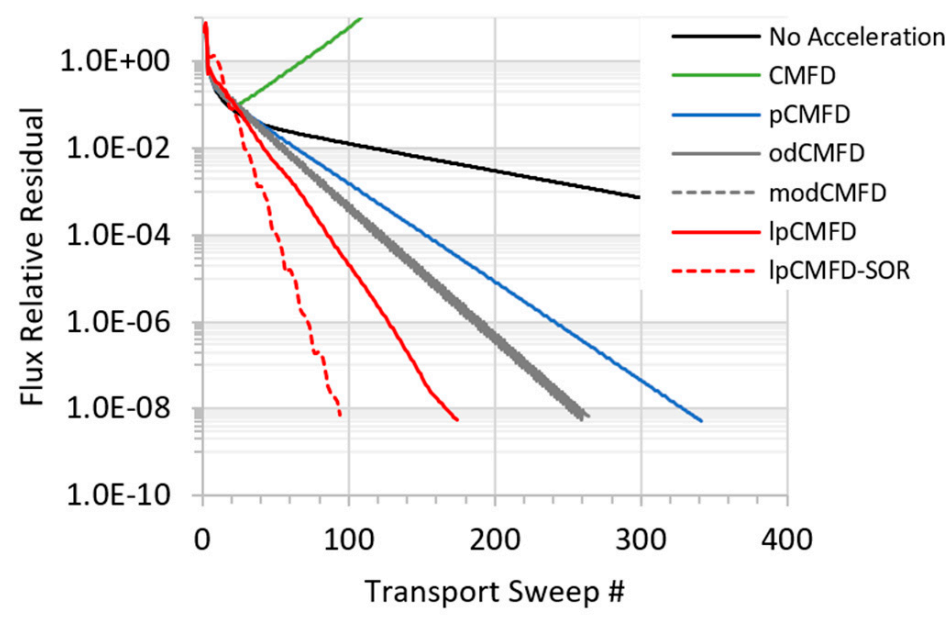

(a)

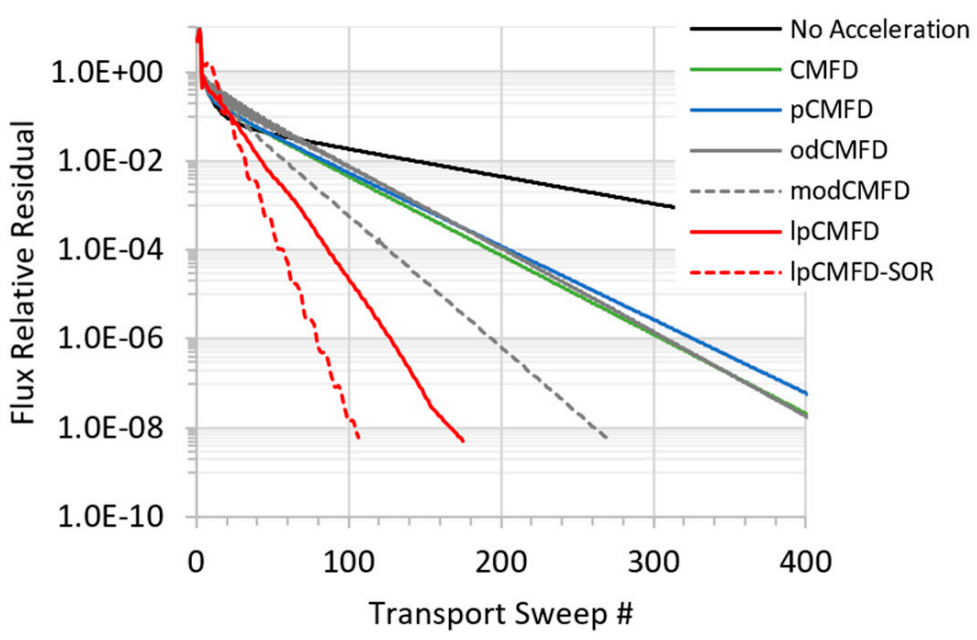

(b)

Figure 9. Results of the 2D test problem. (a) Case A $(c=0.9)$; (b) Case B $(c=0.7)$.

\subsection{Two-Dimensional Mini-Assembly Problem}

In this section, the convergence comparison of the CMFD schemes for a $2 \mathrm{D}$ miniassembly k-eigenvalue problem is shown in Figure 10. Similar to the previous problem, the problem domain is a $6 \times 6 \mathrm{~cm}$ square with the reflective boundary condition on each side. The domain is divided into $5 \times 5$ uniform coarse-mesh cells, and each coarse cell consists of $10 \times 10$ fine-mesh cells. In each coarse cell, the central $4 \times 4$ fine cells represent the fuel region, and all the other cells are the water region. The numerical solutions for the $S_{N}$ transport are obtained on the fine mesh $(50 \times 50)$ using the level-symmetric $S_{12}$ quadrature set for angular discretization and the DD method for spatial discretization. The CMFD solutions are obtained on the coarse-mesh grids $(5 \times 5)$. The homogenized cross-sections on the coarse-mesh cells are updated during each iteration by weighting with the volume and flux. 


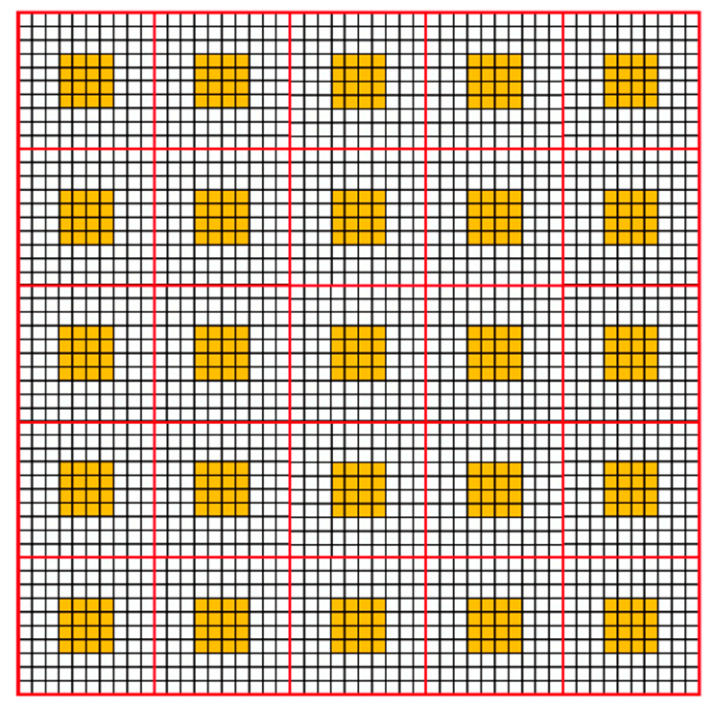

$$
\begin{aligned}
& \square \Sigma_{t}=3 \mathrm{~cm}^{-1}, c=0.995, v \Sigma_{f}=0 \mathrm{~cm}^{-1} \\
& \square \Sigma_{t}=1 \mathrm{~cm}^{-1}, c=0.6, v \Sigma_{f}=0.5 \mathrm{~cm}^{-1}
\end{aligned}
$$

Figure 10. 2D mini-assembly problem.

The normalized scalar flux is plotted in Figure 11a. The convergence comparison is illustrated in Figure 11b. In this problem, CMFD also fails to converge. The convergence of odCMFD is similar to that of modCMFD. Again, the lpCMFD scheme outperforms other schemes, and SOR can improve the convergence rate significantly.

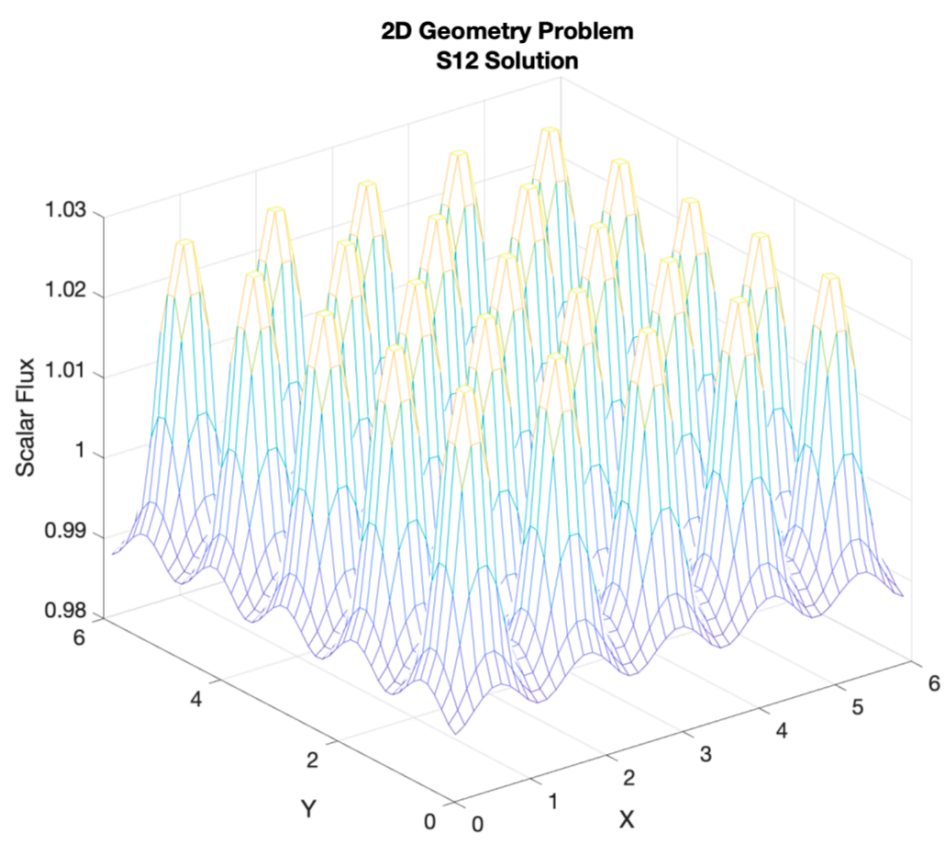

(a)

Figure 11. Cont. 


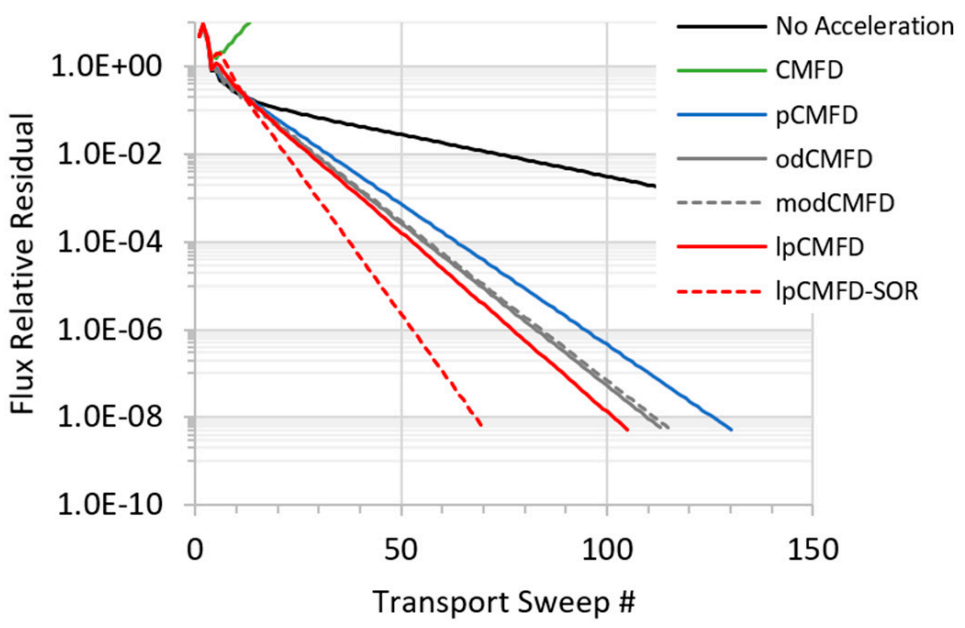

(b)

Figure 11. Results of the 2D mini-assembly problem. (a) Converged scalar flux; (b) convergence comparison.

\section{Conclusions}

Fourier analysis results have been presented for the acceleration schemes: CMFD, pCMFD, odCMFD, and lpCMFD. It has been shown that the DD transport solver predicts slightly larger spectral radii than the SC, which can have some implications on the development of diffusion-based stabilizing techniques, as exemplified by the odCMFD scheme. The odCMFD becomes less effective and unstable for a relatively large optical thickness in the k-eigenvalue problems. This is because the original optimal artificial diffusion coefficients determined by the SC solution are less sufficient for stability. To stabilize the odCMFD, these coefficients have been redeveloped based on the DD-based Fourier analysis. The numerical results for different test problems have confirmed that the modified odCMFD (modCMFD) scheme is more stable and effective.

The lpCMFD convergence can be enhanced with SOR. However, finding an optimal global SOR is not straightforward and is problem-dependent. In this paper, two explicit expressions have been proposed for the calculation of local optimal SOR factors-one is for fixed source problems, and the other is for k-eigenvalue problems. These local SOR factors can be precomputed or updated on-the-fly during the iteration.

Author Contributions: Conceptualization, D.W.; methodology, D.W.; formal analysis, D.W. and Z.Z.; writing — original draft preparation, D.W.; writing — review and editing, D.W. and Z.Z.; supervision, D.W.; project administration, D.W.; funding acquisition, D.W. All authors have read and agreed to the published version of the manuscript.

Funding: This research received no external funding.

Conflicts of Interest: The authors declare no conflict of interest.

\section{Appendix A. CMFD Algorithm and Formulation}

The coupled iteration procedure for the k-eigenvalue case is described as follows. Note that the algorithm for the fixed-source case follows a similar procedure, which is omitted for brevity. To facilitate the discussion, the model problem is a slab geometry, homogeneous monoenergetic problem with isotropic scattering and periodic boundary conditions. The model problem is solved with the $S_{N}$ transport equation coupled with a CMFD-based acceleration scheme. 


\section{Perform a transport sweep:}

$$
\mu_{n} \frac{\partial \psi_{n}^{(l+1 / 2)}}{\partial x}+\Sigma_{t}(x) \psi_{n}^{(l+1 / 2)}=\frac{1}{2}\left[\Sigma_{s}(x)+\lambda^{(l)} v \Sigma_{f}(x)\right] \phi^{(l)}(x),
$$

where

$\mu_{n}=$ neutron direction cosine with respect to $x$;

$\psi_{n}=$ angular flux;

$\phi=\sum_{n=1}^{N} \psi_{n} w_{n}$, scalar flux;

$w_{n}=$ quadrature weights;

$\Sigma_{t}=$ total macroscopic cross-section;

$\Sigma_{\mathrm{s}}=$ macroscopic scattering cross-section;

$v \Sigma_{f}=$ macroscopic nu-fission cross-section;

$\lambda=\frac{1}{k}$, where $k$ is the neutron multiplication factor;

$l=$ iteration index.

2. Compute the cross-sections, the diffusion coefficient, and the nonlinear current correction factor for the low-order diffusion equation:

The coarse-mesh cross-sections are calculated by flux weighting as

$$
\Sigma_{i, C M}^{(l+1 / 2)}=\frac{\int_{\Delta} \Sigma_{i}(x) \phi^{(l+1 / 2)}(x) d x}{\int_{\Delta} \phi^{(l+1 / 2)}(x) d x}
$$

where $i=t, a, s, f$ for total, absorption, scattering, and fission macroscopic cross-sections, respectively. $\Delta$ is the coarse-mesh cell size. The diffusion coefficient and the nonlinear current correction factor are then defined as

$$
\begin{gathered}
D_{C M}^{(l+1 / 2)}=\frac{1}{3 \Sigma_{t, C M}^{(l+1 / 2)}}, \\
\hat{D}_{C M}^{(l+1 / 2)}=\frac{J^{(l+1 / 2)}+D_{C M}^{(l+1 / 2)} \frac{d \bar{\phi}^{(l+1 / 2)}}{d x}}{\bar{\phi}^{(l+1 / 2)}}
\end{gathered}
$$

3. Solve the low-order diffusion eigenvalue problem using the power iteration:

$$
\frac{d}{d x}\left[-D_{C M}^{(l+1 / 2)} \frac{d}{d x}+\hat{D}_{C M}^{(l+1 / 2)}\right] \Phi^{(l+1, k+1)}(x)+\Sigma_{a, C M}^{(l+1 / 2)} \Phi^{(l+1, k+1)}=\lambda^{(l+1, k+1)} v \Sigma_{f, C M}^{(l+1 / 2)} \Phi^{(l+1, k)}(x),
$$

where is the power iteration index.

4. Update the scalar flux:

$$
\phi^{(n+1)}(x)=\phi^{(n+1 / 2)}(x)+\delta\left[\Phi^{(n+1)}, \bar{\phi}^{(n+1 / 2)}, \phi^{(n+1 / 2)}\right],
$$

where $\delta$ is a general representation of different CMFD schemes. For the standard CMFD scheme, it is given as

$$
\delta=\left(\frac{\Phi^{(n+1)}}{\bar{\phi}^{(n+1 / 2)}}-1\right) \phi^{(n+1 / 2)} .
$$

The above $S_{N}$ equation (Equation (A1)) and the diffusion equation (Equation (A5)) can be solved using various discretization schemes. In Fourier analysis, the $\mathrm{S}_{N}$ equation is typically discretized using the SC or DD method on a fine mesh grid, as shown in Figure A1. 


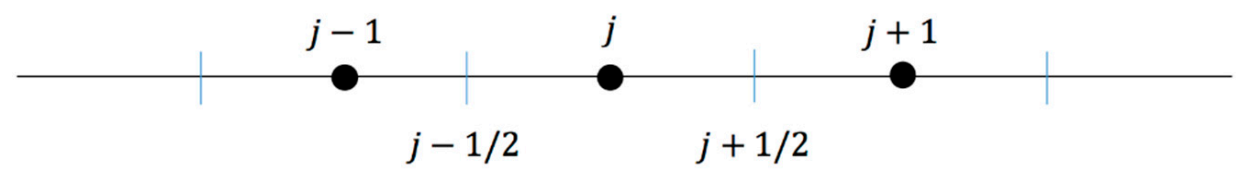

Figure A1. One-dimensional mesh.

$$
\frac{\mu_{n}}{h_{j}}\left(\psi_{n, j+1 / 2}^{l+1 / 2}-\psi_{n, j-1 / 2}^{l+1 / 2}\right)+\Sigma_{t, j} \psi_{n, j}^{l+1 / 2}=\frac{1}{2}\left[\Sigma_{s, j}+\lambda^{(l)} v \Sigma_{f, j}\right] \phi_{j}^{(l)},
$$

where

$$
\begin{gathered}
\psi_{n, j}=\left(\frac{1-\alpha_{n, j}}{2}\right) \psi_{n, j-1 / 2}+\left(\frac{1+\alpha_{n, j}}{2}\right) \psi_{n, j+1 / 2} \\
\alpha_{n, j}=\left\{\begin{array}{cc}
\frac{1+e^{-\Sigma_{t, j} h_{j} / \mu_{n}}}{1-e^{-\Sigma_{t, j} h_{j} / \mu_{n}}-\frac{2 \mu_{n}}{\Sigma_{t, j} h_{j}}}, & S C \\
0, & D D
\end{array}\right.
\end{gathered}
$$

$h_{j}$ mesh size of fine-mesh cell $j$.

The diffusion equation (Equation (A5)) is solved on a coarse-mesh grid, where each coarse cell consists of $p$ fine-mesh cells.

$$
\begin{gathered}
\frac{-\left(D_{C M, i}^{(l+1 / 2)}+D_{C M, i+1}^{(l+1 / 2)}\right)\left(\Phi_{i+1}^{(l+1, k+1)}-\Phi_{i}^{(l+1, k+1)}\right)}{2 h_{C M}^{2}}+\frac{\left(D_{C M, i}^{(l+1 / 2)}+D_{C M, i-1}^{(l+1 / 2)}\right)\left(\Phi_{i}^{(l+1, k+1)}-\Phi_{i-1}^{(l+1, k+1)}\right)}{2 h_{C M}^{2}}+ \\
\frac{\hat{D}_{C M, i+1 / 2}^{(l+1 / 2)}\left(\Phi_{i+1}^{(l+1, k+1)}+\Phi_{i}^{(l+1, k+1)}\right)-\hat{D}_{C M, i-1 / 2}^{(l+1 / 2)}\left(\Phi_{i}^{(l+1, k+1)}+\Phi_{i-1}^{(l+1, k+1)}\right)}{h_{C M}}+\Sigma_{a, C M, i} \Phi_{i}^{(l+1, k+1)} \\
=\lambda^{(l+1, k+1)} v \Sigma_{f, C M, i}^{(l+1 / 2)} \Phi_{i}^{(l+1, k)}
\end{gathered}
$$

where $i$ is the coarse-mesh cell index. $h_{C M}$ is the coarse-mesh cell size.

\section{References}

1. Smith, K.S. Nodal Method Storage Reduction by Non-Linear Iteration. Trans. Am. Nucl. Soc. 1984, 44, 265.

2. Smith, K.S.; Rhodes, J.D., III. CASMO Characteristics Methods for Two-Dimensional PWR and BWR Core Calculations. Trans. Am. Nucl. Soc. 2000, 83, 294.

3. Cho, N.Z.; Park, C.J. A Comparison of Coarse Mesh Rebalance and Coarse Mesh Finite Difference Accelerations for the Neutron Transport Calculations. In Proceedings of the M\&C 2003, Gatlinburg, TN, USA, 6-11 April 2003. Available online: https://github.com/nzcho/Nurapt-Archives/blob/master/NurapT2002_2rev2.pdf (accessed on 13 January 2021).

4. Li, L.; Smith, K.S.; Forget, B. Techniques for stabilizing Coarse-Mesh Finite Difference (CMFD) in method of characteristics (MOC). In Proceedings of the Joint International Conference on Mathematics and Computation (M\&C), Supercomputing in Nuclear Applications (SNA) and the Monte Carlo (MC) Method, ANS MC2015, Nashville, TN, USA, 19-23 April 2015; American Nuclear Society: La Grange Park, IL, USA, 2015.

5. Keady, K.P.; Larsen, E.W. Stability of Sn K-Eigenvalue Iterations Using CMFD Acceleration. In Proceedings of the Joint International Conference on Mathematics and Computation (M\&C), Supercomputing in Nuclear Applications (SNA) and the Monte Carlo (MC) Method, ANS MC2015, Nashville, TN, USA, 19-23 April 2015; American Nuclear Society: La Grange Park, IL, USA, 2015.

6. Jarret, M.; Kochunas, B.; Zhu, A.; Downar, T. Analysis of Stabilization Techniques for CMFD Acceleration of Neutron Transport Problems. Nucl. Sci. Eng. 2016, 184, 208-227. [CrossRef]

7. Zhu, A.; Jarrett, M.; Xu, Y.; Kochunas, B.; Larsen, E.; Downar, T. An optimally diffusive coarse mesh finite difference method to accelerate neutron transport calculations. Ann. Nucl. Energy 2016, 95, 116-124. [CrossRef]

8. Wang, D.; Xiao, S. A Linear Prolongation Approach to Stabilizing CMFD. Nucl. Sci. Eng. 2018, 190, 45-55. [CrossRef]

9. Wang, D.; Xiao, S.; Xu, Y.; Downar, T.; Shemon, E.; Xing, Y. Stabilizing CMFD with Linear Prolongation. In Proceedings of the PHYSOR 2018, Cancun, Mexico, 22-26 April 2018.

10. Shen, Q.; Xu, Y.; Downar, T.; Wang, D. Stability Analysis of lpCMFD for Accelerating $S_{N}$ Method. In Proceedings of the PHYSOR 2018, Cancun, Mexico, 22-26 April 2018. 
11. Shen, Q.; Xu, Y.; Downar, T. Stability analysis of the CMFD scheme with linear prolongation. Ann. Nucl. Energy 2019, 129, 298-307. [CrossRef]

12. Byambaakhuu, T.; Wang, D. Comparison of lpCMFD with Other CMFD Based Acceleration Schemes. In Proceedings of the 2019 ANS Student Conference, Richmond, VA, USA, 4-6 April 2019.

13. Li, J.; Xu, Y.; Wang, D.; Shen, Q.; Kochunas, B.; Downar, T. Demonstration of a Linear Prolongation CMFD Method on MOC. In Proceedings of the PHYSOR 2020, Cambridge, UK, 29 March-2 April 2020.

14. Oshima, Y.; Endo, T.; Yamamoto, A.; Kodama, Y.; Ohoka, Y.; Nagano, H. Impact of Various Parameters on Convergence Performance of CMFD Acceleration for MOC in Multigroup Heterogeneous Geometry. Nucl. Sci. Eng. 2020, 194, 477-491. [CrossRef]

15. Wang, D. Enhancing lpCMFD Acceleration with Successive Overrelaxation for Neutron Transport Source Iteration. Nucl. Sci. Eng. 2021, 195, 1-12. [CrossRef]

16. Wang, D. Application of lpCMFD for K-Eigenvalue Transport Problems with Feedback. Trans. Am. Nucl. Soc. 2020, 123, 681-684. 